\title{
Reference Gene Validation Within the Rat Brain Under Mild Intermittent Ketosis Induced by Supplementation with Medium-Chain Triglycerides
}

\section{Alexander Pavlovich Schwarz}

Institute of Evolutionary Physiology and Biochemistry

Veronika Alexandrovna Nikitina

Institute of Experimental Medicine

Darya Uladzimirawna Krytskaya

Institute of Experimental Medicine

Ksenia Pavlovna Shcherbakova

Institute of Experimental Medicine

Alexander Nikolaevich Trofimov ( $\nabla$ alexander.n.trofimov@gmail.com )

Institute of Experimental Medicine

Research Article

Keywords: RT-qPCR, mRNA, mild ketosis, medium-chain triglycerides, RefFinder ${ }^{\circledR}$, hippocampus

Posted Date: September 2nd, 2021

DOl: https://doi.org/10.21203/rs.3.rs-851204/v1

License: (c) (i) This work is licensed under a Creative Commons Attribution 4.0 International License.

Read Full License 


\section{Abstract}

RT-qPCR has become the gold standard in mRNA expression analysis. However, it requires an accurate choice of reference genes for adequate normalization. This work aims to validate the reference genes for qPCR experiments in the brain of rats in the model of mild ketosis established through supplementation with medium-chain triglycerides (MCT) and intermittent fasting. The standard chow of adult Wistar rats was supplemented with MCT oil ( $2 \mathrm{ml} / \mathrm{kg}$, i.g., during $6 \mathrm{~h}$ of fasting) or water (equivolume) for 1 month. The mRNA expression of 9 housekeeping genes (Actb, B2m, Gapdh, Hprt1, Pgk1, Ppia, Rpl13a, Sdha, $Y$ whaz) in the medial prefrontal cortex, dorsal and ventral hippocampus was measured by RT-qPCR. Using the RefFinder ${ }^{\circledR}$ online tool, we have found that the reference gene stability ranking strongly depended on the analyzed brain region. The most stably expressed reference genes were found to be Ppia, Actb, and Rp/13a in the medial prefrontal cortex; Rp/13a, Ywhaz, and Pgk1 in the dorsal hippocampus; Ywhaz, Sdha, and Ppia in the ventral hippocampus. The B2m was identified as an invalid reference gene in the ventral hippocampus, while Sdha, Actb, and Gapdh were unstable in the dorsal hippocampus. The stabilities of the examined housekeeping genes were lower in the dorsal hippocampus compared to the ventral hippocampus and the medial prefrontal cortex. Thus, the expression stability of reference genes strongly depends on the examined brain regions. The dorsal and ventral hippocampal areas differ in reference genes stability rankings, which should be taken into account in the RT-qPCR experimental design.

\section{Introduction}

Reverse transcription followed by quantitative (real-time) polymerase chain reaction (RT-qPCR) is known to be a gold standard in low throughput mRNA expression analysis. However, it requires an accurate choice of reference genes for normalization to obtain reliable results. Recent findings show that reference gene stability may be affected by experimental design ${ }^{1}$. The choice of an unstable reference gene for RTqPCR data normalization could conceal changes of target mRNA levels or erroneously reveal irrelevant effects of experimental treatments ${ }^{2-8}$.

In the present work, we aim to evaluate the expression stability of 9 housekeeping genes widely used as reference and select suitable reference genes for RT-qPCR analysis within the prefrontal cortex and hippocampus in the rat model of mild ketosis induced by medium-chain triglyceride supplementation. MCT supplementation is considered a promising approach to mimic the neuroprotective effects of the classic ketogenic diet without its adverse effects ${ }^{9,10}$ and has been shown to affect the expression of neuroplasticity-related genes ${ }^{11}$ and exert effects in the brain in a region-specific manner ${ }^{12}$.

Gapdh, Actb, Ppia, B2m, Hprt1, Ywhaz, Rp/13a, Pgk1, Sdha genes selected for analysis are widely used as reference in RT-qPCR experiments in brain tissues or brain-derived cell lines in laboratory rats and have shown higher or lower stability depending on experimental settings ${ }^{1}$.

\section{Results}


The expression stability of nine reference genes (Actb, B2m, Gapdh, Hprt1, Pgk1, Ppia, Rp/13a, Sdha, $Y w h a z)$ in several brain regions (medial prefrontal cortex, dorsal and ventral hippocampus) of rats in the model of mild ketosis was analyzed by RefFinder ${ }^{\circledR}$ online tool (https://www.heartcure.com.au/reffinder/) incorporating comparative Delta-Ct ${ }^{13}$, BestKeeper ${ }^{14}$, NormFinder ${ }^{15}$, and GeNorm ${ }^{16}$ algorithms. Stability values and rankings obtained by different methods are summarized in Figs. 1 and 2, respectively. Detailed results are provided in supplementary files (Figures S1-S3). As can be seen in Fig. 1, the stability values among the examined brain areas are proportionally quite similar regardless of the algorithm chosen for the analysis. Overall, the examined reference genes demonstrated the highest stability in the medial prefrontal cortex and the lowest stability in the dorsal hippocampus (Fig. 1).

\section{Medial Prefrontal cortex}

Ppia, Actb, and Rp/13a were found to be the most stable reference genes within the medial prefrontal cortex of rats subjected to mild ketosis, based on RefFinder comprehensive ranking (Fig. 2A). The B2m expression was the least stable. GeNorm identified Actb and Gapdh as the most stably expressed reference genes, while $B 2 m$ and Sdha were identified as the least stable. GeNorm M-values for all genes were below 0.5 cut-off value. NormFinder found Ppia and Rp/13a to be the most stable, whereas $B 2 m$ and Sdha were the least stable. According to the comparative Delta-Ct method, Ppia, Rp/13a, and Actb were the most stable, and $B 2 m$ and Sdha were the least stable. BestKeeper identified Sdha, Pgk1, and Ywhaz as the most stably expressed, and $B 2 m$ as the least stably expressed.

\section{Dorsal hippocampus}

RefFinder comprehensive ranking identified $R p / 13 a, Y w h a z, P g k 1$ as the most stable reference genes in the dorsal hippocampus, whereas Sdha expression was the least stable (Fig. 2B). Rp/13a and Ywhaz were found to be the most stable according to GeNorm analysis, and Gapdh, Actb, and Sdha were identified as unstable and invalid reference genes ( 0.5 cut-off M-value). Rp/13a and Ywhaz were also found to be the most stable reference genes by NormFinder and comparative Delta-Ct algorithms, whereas $A c t b$ and Sdha expression was the least stable based on these methods. BestKeeper identified Pgk1 and Rp/13a as the most stable, and $B 2 m$ as the least stable.

\section{Ventral hippocampus}

Ywhaz, Sdha, and Ppia were the most stably expressed in the ventral hippocampus, whereas Gapdh and $B 2 m$ were the most unstable, based on RefFinder comprehensive ranking (Fig. 2C). Ywhaz, Sdha, and $R p / 13 a$ were found to be the most stable, while Gapdh and B2m were found to be the least stable reference genes according to NormFinder and comparative Delta-Ct results. Best Keeper ranked Actb, $P g k 1$, and Sdha as the most stable, and B2m as the least stable reference gene. Ppia and Hprt1 were the best choice reference genes according to the GeNorm algorithm. $B 2 m$ expression was unstable and therefore would be invalid for RT-qPCR data normalization according to the GeNorm 0.5 cut-off M-Value.

\section{Discussion}


In the present work, we have analyzed the expression stability of nine housekeeping genes within the rat brain in the model of mild ketosis induced by MCT supplementation and intermittent fasting. We found that stability rankings strongly depended on the analyzed brain region.

The reference gene stability rankings significantly differed in the dorsal and ventral hippocampal areas. For example, Sdha expression was one of the most stable in the ventral hippocampus, while in the dorsal hippocampus it had low stability and is therefore inappropriate for RT-qPCR data normalization. It is well known that dorsal and ventral hippocampal areas have different transcriptomic and functional properties 17. Our present work as well as our recent study in the model of juvenile seizures ${ }^{18}$ suggest that reference gene stability varies in different hippocampal areas, which should be taken into account during RT-qPCR experimental design. Our findings on the heterogeneity of housekeeping gene expression also emphasize that dorsal and ventral parts of the hippocampus should be analyzed separately in biochemical experiments.

The examined reference gene panel, overall, demonstrated lower stability in the dorsal hippocampus compared to the ventral hippocampus and the medial prefrontal cortex (Fig. 1). This may reflect a higher sensitivity of dorsal hippocampus to ketogenic treatment. Our data are in agreement with a wellestablished fact that ketogenic diet and MCT supplementation improve dorsal hippocampus-dependent cognitive functions ${ }^{11,19}$. It should be noted that the protein products encoded by two of the three housekeeping genes with unstable expression in the dorsal hippocampus participate in glucose (glyceraldehyde-3-phosphate dehydrogenase) and energy (succinate dehydrogenase subunit A) metabolism, which are known to be affected by ketogenic diet ${ }^{19}$.

To sum up, we can conclude that reference gene expression stability varies among brain areas. The housekeeping gene expression seems to be affected by MCT supplementation in the dorsal hippocampus to a greater extent than in the ventral hippocampus and medial prefrontal cortex.

\section{Methods}

The study was performed on 9 m.o. male Wistar rats weighed $444 \pm 69 \mathrm{~g}(\mathrm{M} \pm \mathrm{SD})$. Animal experiments were carried out under the Guidelines on the Treatment of Laboratory Animals effective at the Institute of Experimental Medicine (St. Petersburg, Russia), and these guidelines comply with EU Directive 2010/63/EU for animal experiments. Experimental protocol was approved by the Ethical committee of the Institute of Experimental Medicine. All procedures reported here were carried out in compliance with the "Animal Research: Reporting of In Vivo Experiments" (ARRIVE) guidelines.

Experimental animals were housed under standard conditions with $12 \mathrm{~h} \mathrm{light-dark} \mathrm{cycle} \mathrm{(lights} \mathrm{on} \mathrm{at} 5$ AM) and ad libitum access to water. Rats were given MCT oil (Jarrow Formulas, Inc., Los Angeles CA, USA) at a dose $2 \mathrm{ml} / \mathrm{kg}$ daily by oral gavage during a 6-hour fasting period or an equivolume of water without fasting (control animals) for 4 weeks. Animals were sacrificed by decapitation. Brains were quickly collected, frozen, and stored at $-70^{\circ} \mathrm{C}$ for further analysis. 
Brains were placed into freezing microtome Thermo-scientific ${ }^{\text {TM }}$ Microm HM525 (Thermo Fisher Scientific, Waltham, MA, USA) for 60 min at $-20^{\circ} \mathrm{C}$ and then cut in coronal direction. Medial prefrontal cortex, dorsal and ventral hippocampal regions were captured by microspatule, the structures determined based on the rat brain atlas ${ }^{20}$, as described earlier ${ }^{18}$. Samples were homogenized in an appropriate volume of ExtractRNA reagent (Evrogen JSC, Moscow, Russia) and total RNA was extracted by single-step acid guanidinium-thiocyanate-phenol-chloroform method ${ }^{21}$ according to the manufacturer's instructions.

To purify the isolated RNA from possible genomic DNA contamination, the samples were treated with ribonuclease-free deoxyribonuclease (RQ1 DNase; Promega Corp., Madison, WI, USA). The tubes with the previously precipitated RNA were centrifuged for $30 \mathrm{~min}$ at $+4^{\circ} \mathrm{C}, 12,000 \mathrm{~g}$, and the ethanol supernatant was removed. The pellet was dried using a dry-block thermostat TS-100 (Biosan, Riga, Latvia) for 5 min at $+50^{\circ} \mathrm{C}$. The dried pellet was resuspended in $7.5 \mu \mathrm{l}$ of milli-Q water and incubated at $+50^{\circ} \mathrm{C}$ for $5 \mathrm{~min}$ for better dissolution of nucleic acids. A reaction mixture containing $10 \mathrm{U}$ of a recombinant RNasin Ribonuclease Inhibitor (Sileks, Moscow, Russia) and $2 \mathrm{U}$ of RQ1 DNase in a $2 \times$ reaction buffer was added to the resulting solution. The tubes with the reaction mixture were incubated for $10 \mathrm{~min}$ at $+37^{\circ} \mathrm{C}$. After incubation, $1.5 \mu \mathrm{L}$ (1/10 of the volume of the reaction mixture) of a stop reagent (20 mM EGTA) was added to the samples, mixed, incubated at $+70^{\circ} \mathrm{C}$ to inactivate the enzyme for $5 \mathrm{~min}$, followed by cooling to $+4^{\circ} \mathrm{C}$.

To purify the resulting preparation from the reaction components, RNA was precipitated: the volume of the solution was brought to $30 \mu \mathrm{L}$ with milli-Q water, $3 \mu \mathrm{L}$ (1/10 of the volume of RNA solution) of $3 \mathrm{M}$ sodium acetate solution, and $75 \mu \mathrm{L}$ ( 2.5 volumes of RNA solution) of $96 \%$ ethanol, thoroughly mixed, and then incubated at $-20^{\circ} \mathrm{C}$ overnight. After that, the tubes were centrifuged, the precipitate was washed with $75 \%$ ethanol and stored under alcohol until further handling.

Before carrying out the reverse transcription (RT) reaction, the tubes with RNA were centrifuged for 30 min at $+4^{\circ} \mathrm{C}, 12,000 \mathrm{~g}$, the RNA sediment was dried, and then dissolved in $15 \mu \mathrm{L}$ of milli-Q water. RNA concentration (via $260 \mathrm{~nm}$ absorption) and purity (260/230 and 260/280 $\mathrm{nm}$ absorption ratios) were measured spectrophotometrically using a Nanodrop 2000 instrument (Thermo Fisher Scientific, Waltham, MA, USA). To carry out the RT reaction, $0.5 \mu \mathrm{g}$ oligo(dT) and $0.25 \mu \mathrm{g}$ random-9-mer primers (BioBeagle, St. Petersburg, Russia) were added to $8 \mu \mathrm{L}$ of the RNA solution containing $1 \mu \mathrm{g}$ of RNA preliminarily equalized in terms of concentration and incubated at $+70^{\circ} \mathrm{C}$ for $5 \mathrm{~min}$. Samples were briefly cooled and centrifuged. Then a reaction mixture containing M-MLV reverse transcriptase (Promega Corp., Madison, WI, USA), a mixture of dNTPs ( 25 mM dATP, 25 mM dCTP, 25 mM dGTP, 25 mM dTTP; Medigen, Novosibirsk, Russia), $5 \times$ M-MLV reaction buffer $(250 \mathrm{mM}$ Tris-HCl, $375 \mathrm{mM} \mathrm{KCl}, 15 \mathrm{mM} \mathrm{MgCl}$, $50 \mathrm{mM}$ DLdithiothreitol, $\mathrm{pH}$ 8.3; Promega Corp., Madison, WI, USA), RNasin in deionized water was added. The samples were thoroughly vortexed and incubated for $2 \mathrm{~h}$ at $+42^{\circ} \mathrm{C}$ for the synthesis of cDNA on the RNA template and for $10 \mathrm{~min}$ at $+65^{\circ} \mathrm{C}$ to stop the reaction through enzyme inactivation. The resulting cDNA solution $(20 \mu \mathrm{L})$ was diluted 5 -fold with deionized water and stored at $-20^{\circ} \mathrm{C}$ until real-time polymerase chain reaction (qPCR) with fluorescent probes (TaqMan technology). 
In the present work, we tested a panel of 9 reference genes that regulate different cell functions and are frequently used for RT-qPCR data normalization in the rat brain ${ }^{1}$. We used three triplex qPCR assays validated in our previous work ${ }^{22}: A c t b+G a p d h+B 2 m ; R p / 13 a+S d h a+P p i a ; H p r t 1+P g k 1+Y w h a z$. The descriptions of the used primer/probes are summarized in supplementary files (Table S1).

The Multiplex qPCR reactions had been optimized and fully described in our previous study ${ }^{22}$. Briefly, the reaction mix contained $1 \mu \mathrm{L}$ of cDNA sample, $0.75 \mathrm{U}$ of TaqM-polymerase (Alkor Bio Group, St. Petersburg, Russia), $200 \mathrm{nM}$ of specific forward and reverse primers, and either $200 \mathrm{nM}$ (for Actb) or 100 $\mathrm{nM}$ (for the rest of the genes) TaqMan probes, $3.5 \mathrm{mM} \mathrm{MgCl}_{2}, 250 \mu \mathrm{M}$ dATP/dTTP/dCTP/dGTP in $10 \mu \mathrm{L}$ total volume of $1 \times$ TaqM-reaction buffer. All oligonucleotides were synthesized by DNA-Synthesis Ltd. (Moscow, Russia). All reactions were duplicated with no reverse transcription and no template control. The reactions were run on $\mathrm{C} 1000$ Touch Thermal Cycler combined with CFX96 Real-Time detection system (Bio-Rad Laboratories, Inc., Hercules, CA, USA). The thermal settings were as follows: 1 cycle at $95^{\circ} \mathrm{C}$ for $15 \mathrm{~min}$ (as recommended by the enzyme manufacturer), 5 cycles (without plate read) with a denaturation step $\left(95^{\circ} \mathrm{C}\right.$ for $\left.5 \mathrm{~s}\right)$ and an annealing/elongation step $\left(60^{\circ} \mathrm{C}\right.$ for $\left.10 \mathrm{~s}\right)$ and then 35 cycles with a denaturation step $\left(95^{\circ} \mathrm{C}\right.$ for $\left.5 \mathrm{~s}\right)$ and an annealing/elongation step $\left(60^{\circ} \mathrm{C}\right.$ for $\left.10 \mathrm{~s}\right)$ followed by fluorescence plate read (about $13 \mathrm{~s}$ ).

The PCR curves were analyzed with the CFX Manager software (Bio-Rad Laboratories, Inc., Hercules, CA, USA): the quantification cycle $(\mathrm{Cq})$ values were determined by setting a single threshold. We then imported the raw mean $\mathrm{Cq}$ data to RefFinder ${ }^{\circledR}$ online tool (https://www.heartcure.com.au/reffinder/) to evaluate the expression stability of the examined genes. RefFinder® utilizes four commonly used algorithms for reference gene expression stability analysis (the comparative Delta-Ct method, BestKeeper, NormFinder, and GeNorm) and calculates comprehensive ranking by geometric averaging of obtained ranks ${ }^{23}$. Briefly, (a) in the analysis by the comparative Delta-Ct method, the selection of the most stable reference genes is achieved by comparing the relative expression of pairs of genes in each sample: genes with the smallest mean standard deviation of delta-Ct are considered the most stable ${ }^{13}$; (b) the BestKeeper tool selects the most stable genes by ranking the geometric means of raw $\mathrm{Cq}$ values of each gene, comparing them in pairs, and calculating the coefficient of variation, the standard deviation of the Cq values (the lower, the more stable), and the correlation coefficient (the higher, the more stable) ${ }^{14}$; (c) NormFinder's principle of operation is based on a mathematical model that calculates the stability value of candidate reference genes by estimating both the total variation of the reference candidate genes and the variation between subgroups of samples in a sample set ${ }^{15}$; (d) the basic principle behind the GeNorm algorithm is to calculate the stability value $\mathrm{M}$ (the lower, the more stable) by averaging the pairwise variations among all candidate genes in all samples ${ }^{16}$.

\section{Declarations}

\section{Author contributions}


Conceptualization, APS; data curation, APS, ANT; formal analysis, APS; funding acquisition, APS, KPS, ANT; investigation, APS, VAN, DUK, ANT; methodology, APS, VAN, DUK, KPS, ANT; project administration, ANT; supervision, ANT; writing - original draft, APS; writing - review and editing, APS, VAN, DUK, KPS, ANT.

Authors declare no conflict of interest.

\section{This work is supported by the Russian Science Foundation, grant no. 19-75-10076.}

\section{References}

1. Chapman, J. R. \& Waldenström, J. With Reference to Reference Genes: A Systematic Review of Endogenous Controls in Gene Expression Studies. PloS one 10, e0141853 (2015).

2. Swijsen, A., Nelissen, K., Janssen, D., Rigo, J.-M. \& Hoogland, G. Validation of reference genes for quantitative real-time PCR studies in the dentate gyrus after experimental febrile seizures. BMC research notes 5, 685 (2012).

3. Zhou, J. et al. Validation of reference genes for quantitative real-time PCR in valproic acid rat models of autism. Molecular biology reports 43, 837-47 (2016).

4. Cook, N. L., Kleinig, T. J., Heuvel, C. V.D. \& Vink, R. Reference genes for normalising gene expression data in collagenase-induced rat intracerebral haemorrhage. BMC Molecular Biology 11, 7 (2010).

5. Chen, G. et al. Validation of reliable reference genes for real-time PCR in human umbilical vein endothelial cells on substrates with different stiffness. PloS one 8, e67360 (2013).

6. Nair, A. R., Smeets, K., Keunen, E., Lee, W. \& Thévenod, F. Renal cells exposed to cadmium in vitro and in vivo: normalizing gene expression data. (2014) doi:10.1002/jat.3047.

7. Crans, R. A. J. et al. The validation of Short Interspersed Nuclear Elements (SINEs) as a RT-qPCR normalization strategy in a rodent model for temporal lobe epilepsy. PloS one 14, e0210567 (2019).

8. Paiva, B., Fraga, L., Meirelles, S., Nardi, N. B. \& Camassola, M. Identi fi cation of suitable reference genes for quantitative gene expression analysis in rat adipose stromal cells induced to trilineage differentiation. Gene(2016) doi:10.1016/j.gene.2016.09.002.

9. Courchesne-Loyer, A. et al. Stimulation of mild, sustained ketonemia by medium-chain triacylglycerols in healthy humans: estimated potential contribution to brain energy metabolism. Nutrition (Burbank, Los Angeles County, Calif.) 29, 635-40 (2013).

10. Fortier, M. et al. A ketogenic drink improves brain energy and some measures of cognition in mild cognitive impairment. Alzheimer's \& dementia: the journal of the Alzheimer's Association 15, 625634 (2019).

11. Wang, D. \& Mitchell, E. S. Cognition and Synaptic-Plasticity Related Changes in Aged Rats Supplemented with 8- and 10-Carbon Medium Chain Triglycerides. PloS one 11, e0160159 (2016).

12. Hollis, F., Mitchell, E. S., Canto, C., Wang, D. \& Sandi, C. Medium chain triglyceride diet reduces anxiety-like behaviors and enhances social competitiveness in rats. Neuropharmacology 138, 245- 
256 (2018).

13. Silver, N., Best, S., Jiang, J. \& Thein, S. L. Selection of housekeeping genes for gene expression studies in human reticulocytes using real-time PCR. BMC molecular biology 7, 33 (2006).

14. Pfaffl, M. W., Tichopad, A., Prgomet, C. \& Neuvians, T. P. Determination of stable housekeeping genes, differentially regulated target genes and sample integrity: BestKeeper-Excel-based tool using pairwise correlations. Biotechnology letters 26, 509-15 (2004).

15. Andersen, C. L., Jensen, J. L. \& Ørntoft, T. F. Normalization of real-time quantitative reverse transcription-PCR data: a model-based variance estimation approach to identify genes suited for normalization, applied to bladder and colon cancer data sets. Cancer research 64, 5245-50 (2004).

16. Vandesompele, J. et al. Accurate normalization of real-time quantitative RT-PCR data by geometric averaging of multiple internal control genes. Genome biology 3, RESEARCH0034 (2002).

17. Fanselow, M. S. \& Dong, H.-W. Are the dorsal and ventral hippocampus functionally distinct structures? Neuron 65, 7-19 (2010).

18. Schwarz, A. P. et al. Reference Gene Validation in the Brain Regions of Young Rats after Pentylenetetrazole-Induced Seizures. Biomedicines 8, (2020).

19. Augustin, K. et al. Mechanisms of action for the medium-chain triglyceride ketogenic diet in neurological and metabolic disorders. The Lancet. Neurology 17, 84-93 (2018).

20. Paxinos, G. \& Watson, C. The Rat Brain in Stereotaxic Coordinates, 6th Edition. (Academic Press, 2007).

21. Chomczynski, P. A reagent for the single-step simultaneous isolation of RNA, DNA and proteins from cell and tissue samples. BioTechniques 15, 532-537 (1993).

22. Schwarz, A. P., Malygina, D. A., Kovalenko, A. A., Trofimov, A. N. \& Zaitsev, A. v. Multiplex qPCR assay for assessment of reference gene expression stability in rat tissues/samples. Molecular and cellular probes 53,101611 (2020).

23. Xie, F., Xiao, P., Chen, D., Xu, L. \& Zhang, B. miRDeepFinder: a miRNA analysis tool for deep sequencing of plant small RNAs. Plant molecular biology 80, 75-84 (2012).

\section{Figures}


a

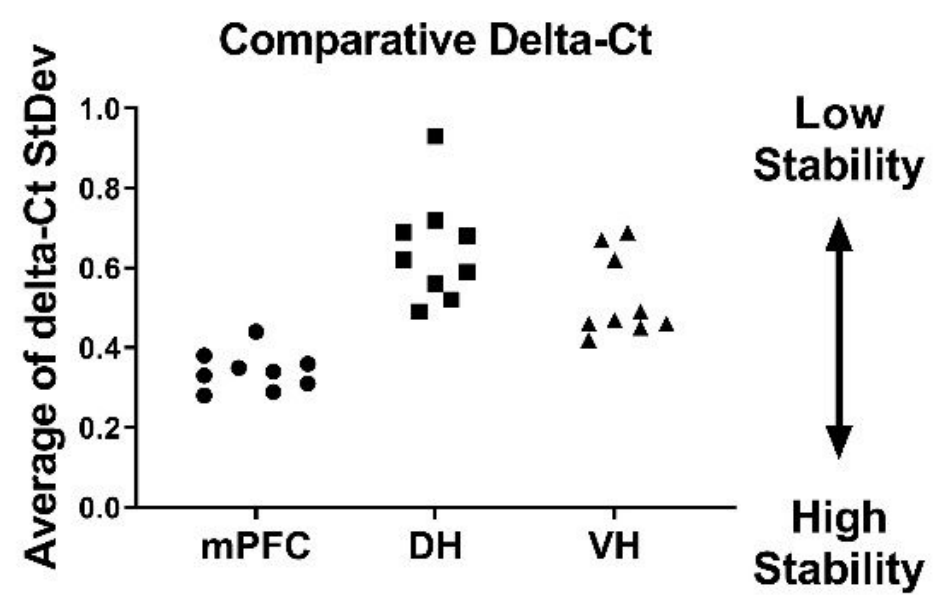

C

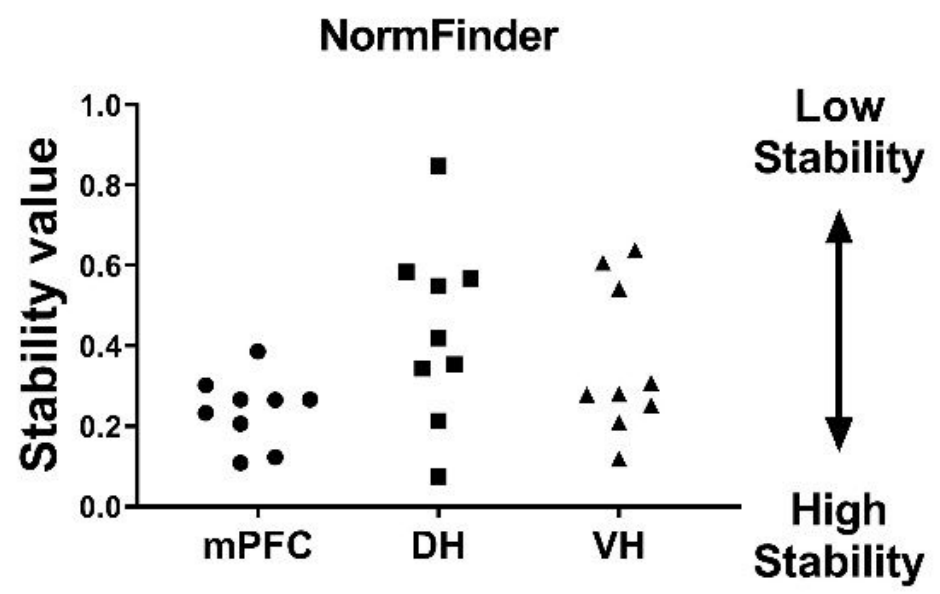

b

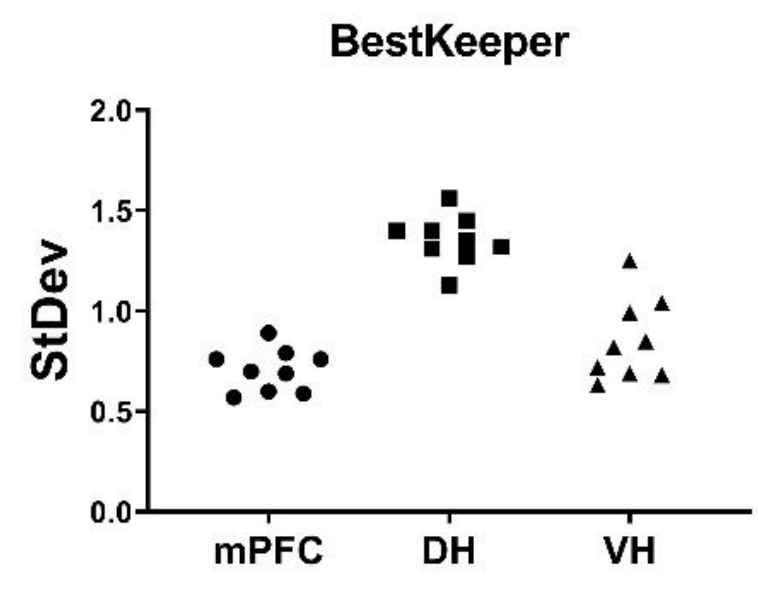

d

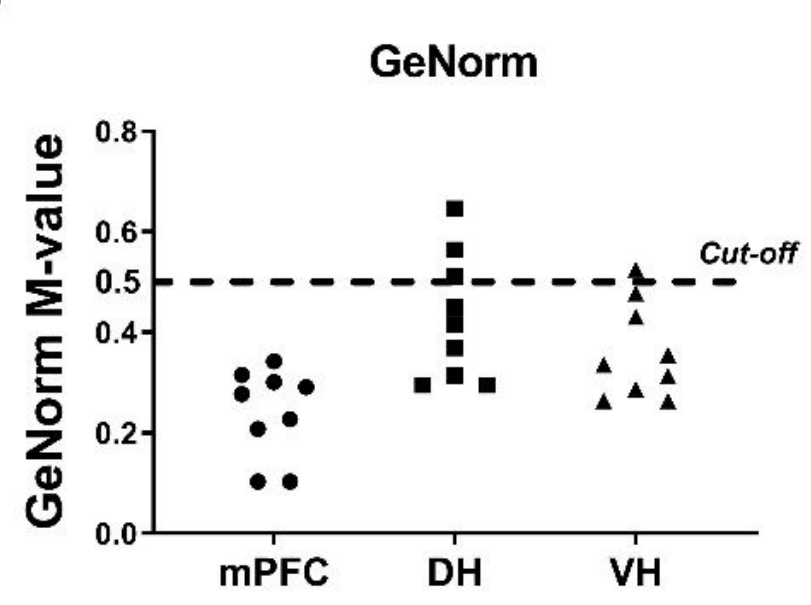

\section{Figure 1}

Reference gene stability values of each housekeeping gene obtained by four methods in three different brain regions of rats fed with MCT. MCT oil ( $2 \mathrm{ml} / \mathrm{kg}$ body weight) was given as a supplement to standard feed to adult ( 9 m.o., BW $=444 \pm 69 \mathrm{~g}$ ) animals by oral gavage daily for 4 weeks, while control rats received equivolume water. Expression stability values of nine housekeeping genes (Actb, Gapdh, B2m, Rpl13a, Sdha, Ppia, Hprt1, Pgk1, Ywhaz, each represented by a dot per each brain structure) in the medial prefrontal cortex (mPFC, circular dots), dorsal ( $\mathrm{DH}$, square dots) and ventral (VH, triangular dots) hippocampal areas were analyzed using (a) comparative Delta-Ct, (b) BestKeeper, (c) NormFinder, and (d) GeNorm methods. For GeNorm (D), values above 0.5 cut-off M-value indicate unstable genes inappropriate for RT-qPCR data normalization. 
a

\section{MPFC}

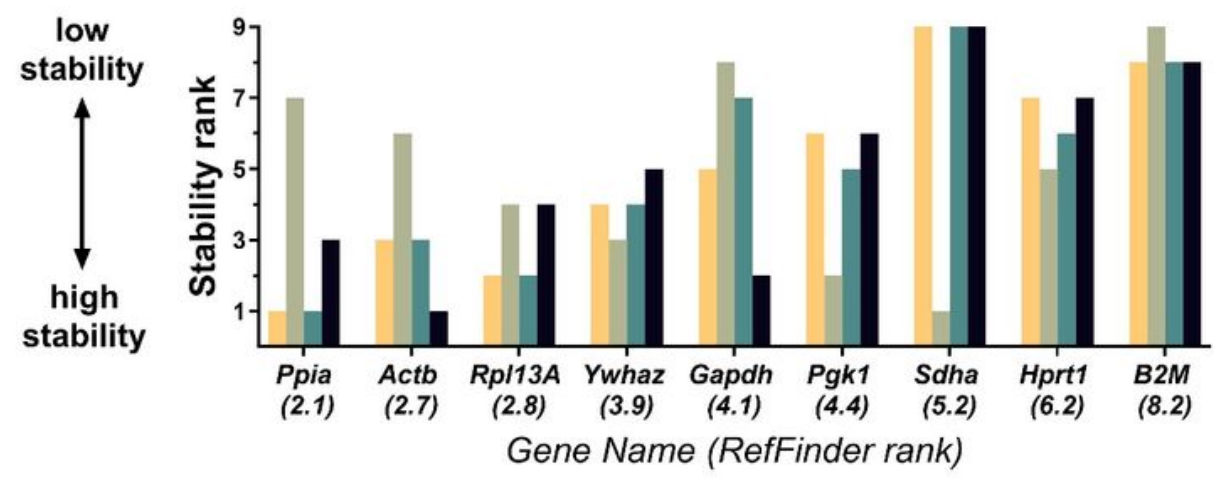

b
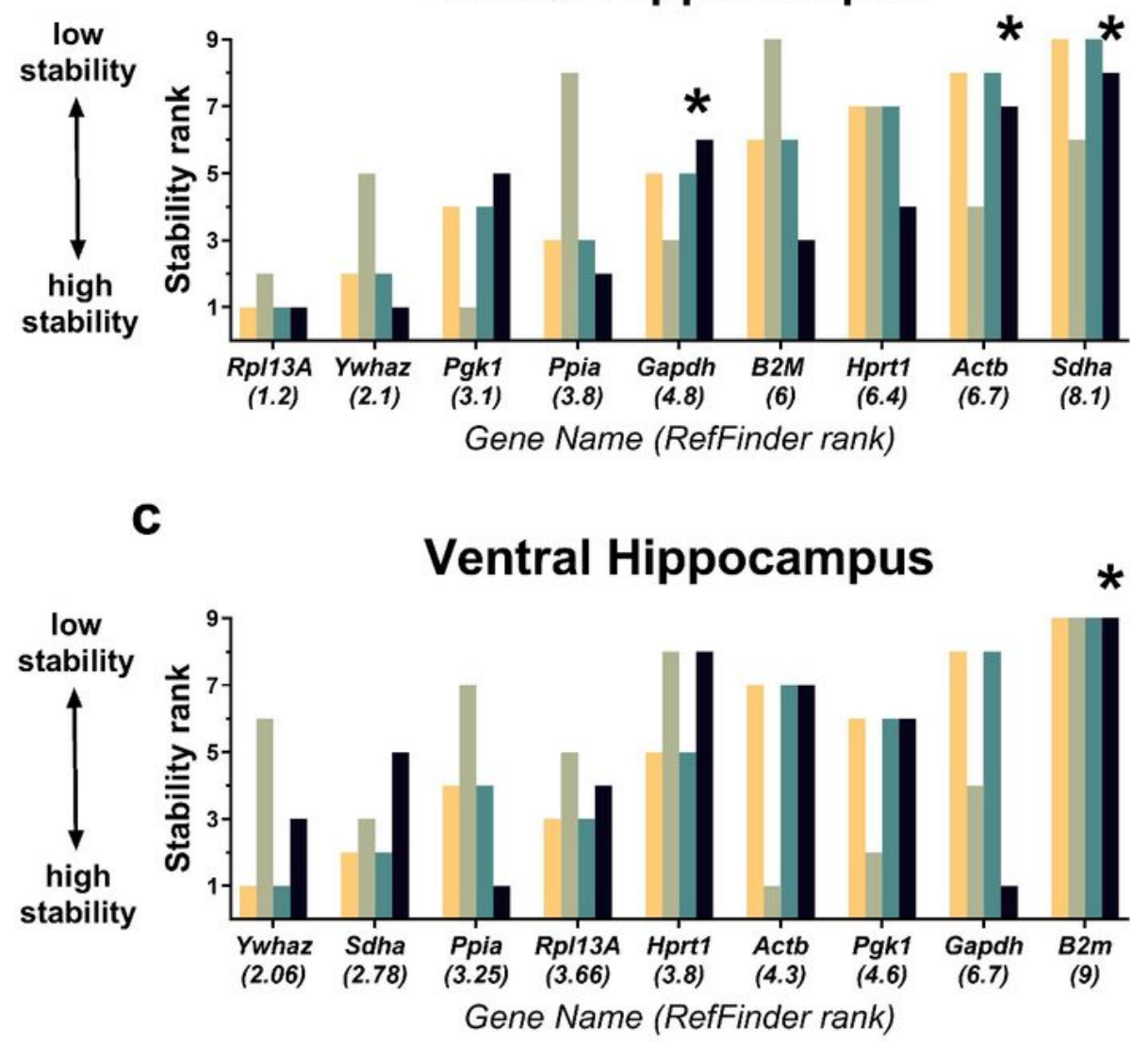

\section{comparative Delta-Ct \\ BestKeeper $\square$ NormFinder $\square$ GeNorm}

Figure 2

Reference gene stability rankings in the three brain regions of rats subjected to medium-chain triglyceride supplementation for 1 month. MCT oil ( $2 \mathrm{ml} / \mathrm{kg}$ body weight) was given as a supplement to standard feed to adult animals ( 9 m.o., BW $=444 \pm 69 \mathrm{~g}$ ) by oral gavage daily for 4 weeks, while control rats received equivolume water. Reference gene stability in the (a) medial prefrontal cortex, (b) dorsal and (c) ventral hippocampal areas was analyzed by RefFinder ${ }^{\circledR}$ online tool. RefFinder comprehensive ranking 
(ranks are indicated in brackets under gene name on X-axis) is based on geometric mean of ranks obtained by four methods: comparative Delta-Ct, BestKeeper, NormFinder, and GeNorm. * - unstably expressed genes inappropriate for normalization, according to the GeNorm cut-off $0.5 \mathrm{M}$-Value.

\section{Supplementary Files}

This is a list of supplementary files associated with this preprint. Click to download.

- 2021.08.27SupplementarymaterialSchwarzetal.toScientificReports.pdf 